\title{
Application of the BOPPPS Instructional Model in Microwave Transmission and Reception Experiments Course
}

\author{
Lili Song, Yang Zhou, Xingfu Gao, Qiang Zhang, Dian Zhang \\ College of Advanced Interdisciplinary Studies, National University of Defense Technology, Changsha, China \\ Email: phoezy@126.com
}

How to cite this paper: Song, L. L., Zhou, Y., Gao, X. F., Zhang, Q., \& Zhang, D. (2020). Application of the BOPPPS Instructional Model in Microwave Transmission and Reception Experiments Course. Open Journal of Social Sciences, 8, 470-476. https://doi.org/10.4236/jss.2020.88040

Received: July 15, 2020

Accepted: August 28, 2020

Published: August 31, 2020

\begin{abstract}
The BOPPPS instructional model is introduced into the experimental course "Microwave Transmission and Reception Experiments". Six pedagogical steps are recommended, that is, Bridge-in, Objective, Pre-assessment, Participatory-learning, Post-assessment and Summary. We aim at improving the teaching skills of the teacher and fostering the abilities and qualities of innovational thinking of the students.
\end{abstract}

\section{Keywords}

BOPPS Instructional Model, Microwave Transmission and Reception

Experiment, Heuristic Education

\section{Introduction to the Teaching Content of "Microwave Transmission and Reception Experiment"}

In the electromagnetic spectrum, the frequency of the microwaves is between ultrashort and infrared waves, which is generally defined as from 1 to $300 \mathrm{GHz}$ (Benford, Swegle, \& Schamiloglu, 2007; Gu \& Zhang, 1989). Microwave has more and more applications in radar communication, advanced material surface treatment, new clean energy, high-energy particle accelerators, advanced food processing, environmental protection, etc. Therefore, it is quite significant to study this experimental course. In addition, this course is highly practical for undergraduate students who major in Optoelectronic Information Science and Engineering. The research content and objectives of this experimental course is as following.

Microwave transmission in space mainly refers to microwaves radiating from an antenna to a region of space and then propagating to the pick-up antenna in some way. The transport medium from the transmitting point to the receiving 
point mainly consists of the atmosphere of the earth and outer space, the ionosphere and the water condensation in the atmosphere. To establish the basic concepts of microwave transmission, we mainly focus on microwave transmission in free space in this course.

The antenna is placed in free space. Assuming that the transmitting antenna is an ideal non-directional antenna, if its radiated power is $P_{\Sigma}$, the power flow density on the sphere away from the antenna $r$ can be expressed as (Kraus \& Marhefka, 2017)

$$
S_{0}=\frac{P_{\Sigma}}{4 \pi r^{2}} \mathrm{~W} / \mathrm{m}^{2}
$$

The power flow density can also be expressed as

$$
S_{0}=\frac{1}{2} \operatorname{Re}\left(E \times H^{*}\right)=\frac{\left|E_{0}\right|^{2}}{240 \pi}
$$

Thus, the value of the electric field intensity $E_{0}$ away from the antenna $r$ is:

$$
E_{0}=\frac{\sqrt{60 P_{\Sigma}}}{r}
$$

In actual life, if its radiation power is $P_{\Sigma}$, assuming that the input power is $P_{i}$ and the gain of the antenna is $G_{i}$, then the value of the electric field intensity $E_{0}$ in the direction of maximum radiation away from the actual antenna $r$ also be modified as:

$$
E_{0}=\frac{\sqrt{60 P_{i} G_{i}}}{r}
$$

Given the gain factor of the pick-up antenna as $G_{r}$, and the area of effective reception as $A_{e}$, the power received by the antenna at $r$ can be expressed as

$$
P_{r}=S_{0} \cdot A_{e}=\frac{P_{i} G_{i}}{4 \pi r^{2}} \cdot \frac{\lambda^{2} G_{r}}{4 \pi}
$$

This formula is a key formula for the design of a wireless telecommunications system, which is also called the Friis transmission formula.

Figure 1 illustrates the experimental schematic. Microwaves with a frequency among 2 to $4 \mathrm{GHz}$ is produced by the signal source, and then transmitted along the coaxial cable to the transmitting antenna and radiated out. After free space transmission of the microwave, the receiving antenna receives the signal and transmits it to the oscilloscope. In order to guarantee the measurement accuracy, the distance between these two antennas must be greater than $0.4 \mathrm{D}^{2} / 1$ (Benford, Swegle, \& Schamiloglu, 2007), where D is the diameter of the horn antenna, 1 is the wavelength of the microwave.

Tow rectangular horn antennas are adopted which are linear polarizations. The model is shown in Figure 2 and the radiation pattern is presented in Figure 3. During the experimental operation, the students need to realize the measurement of received microwave under the condition of modulating the frequency of source microwave, the distance and angle of two antennas. 


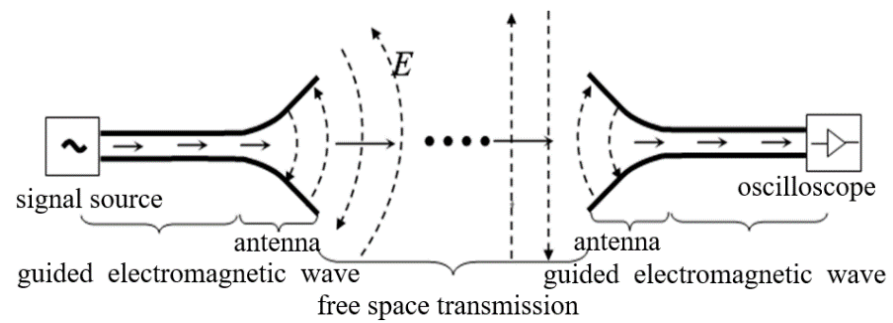

Figure 1. The experimental schematic.

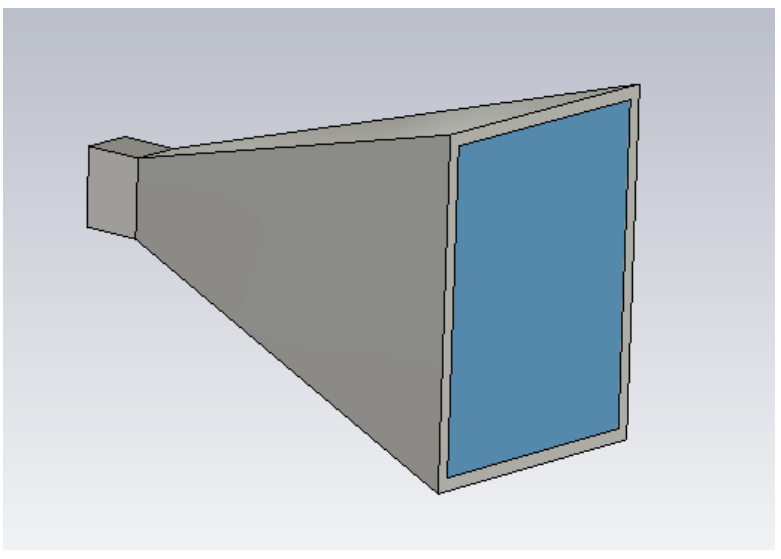

Figure 2. Schematic diagram of the linear polarization antenna.

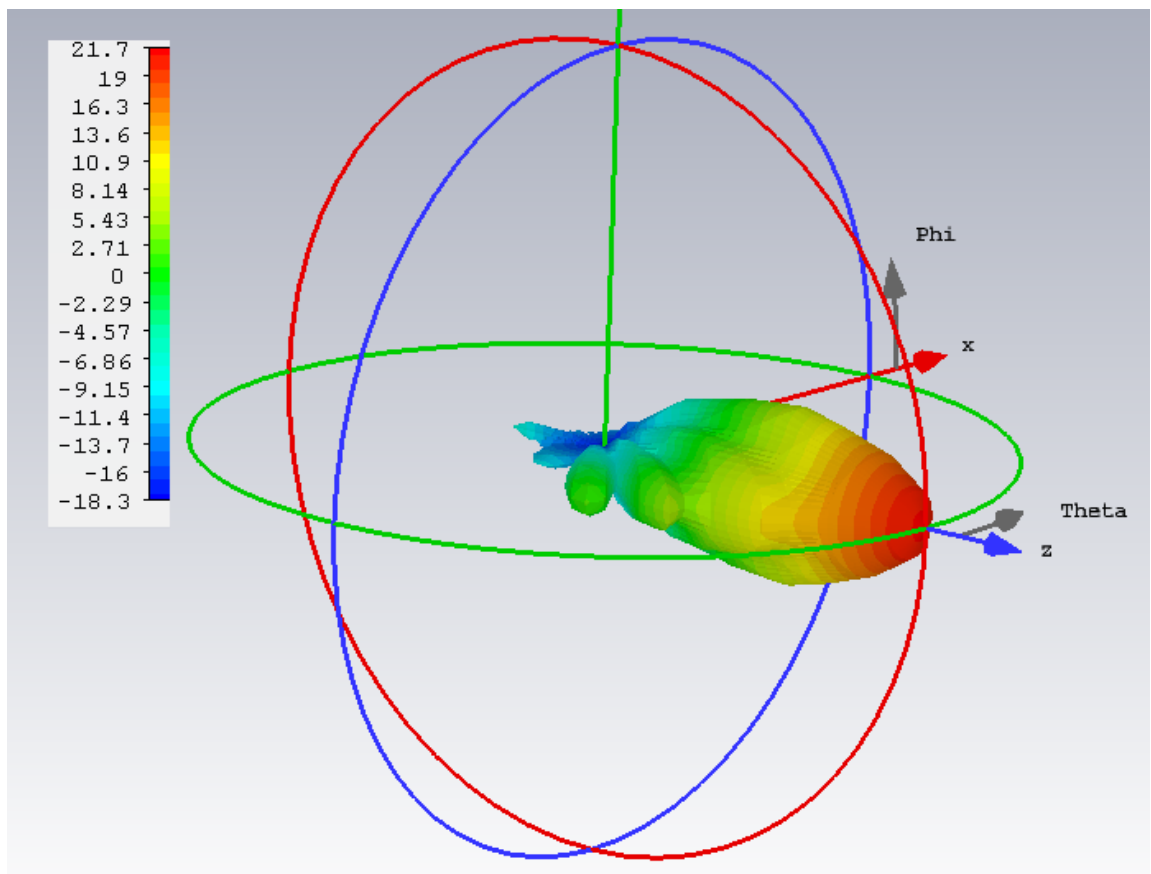

Figure 3. Radiation pattern of the linear polarization antenna.

The class lasts 90 minutes, including approximately 25 minutes to explain research content, and 15 minutes to explain experimental operations, and the rest 50 minutes for students to accomplish the experiment. Approximation, equivalence, empirical formulas, experimental and other practical engineering methods 
are adopted in the course, so as to foster their ability of analyzing and solving practical problems, thus lay a firm foundation for future work in microwave technology research, microwave communication engineering design, photoelectric information system research, high power microwave research and applications.

The introduction of BOPPPS instructional model was investigated, with the objective of achieving better educational performance in this experimental course.

\section{Introduction to the BOPPPS Instructional Model}

The BOPPPS instructional model derives from the Canadian Teacher Skills Training (ISW), which is a goal-oriented, student-centered instructional model. It consists of six instructional components: introduction (Bridge-in), learning objectives (Objective/Outcome), pre-assessment, participatory-learning, post-assessment, and summary.

The fundamental difference between the BOPPPS model and traditional instructional design is as following. The BOPPPS model emphasizes on participatory learning, active role of learners, and making adjustments through continuous feedback on instruction.

\section{Design of the Teaching Skills of "Microwave Transmission and Reception Experiment"}

1) Bridge-in

In bridge-in, skills like asking questions, telling stories, showing videos, playing games and case studies can be adopted. The class will begin with an introduction to the television antenna, which is very common in life, so as to attract their attention. In cable television systems, the antenna receives the high-frequency electromagnetic energy from the television station. The oldest television antenna and Yagi antenna in the 1980s are linear antennas, while satellite pick-up antennas are plane antennas, while satellite pick-up antennas are plane antennasas shown in Figure 4.

By introducing common antennas in daily life, the teaching becomes more attractive. First of all, the images of the antennas are vivid and understandable. Secondly, the sense of era of TV antennas can arouse more interest of students, which can make them immerse in the knowledge more quickly.

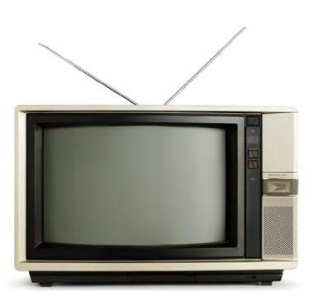

(1) The oldest TV antenna

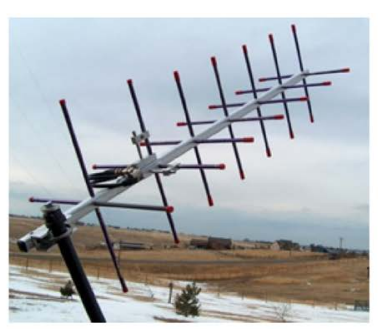

(2) Yagi antenna

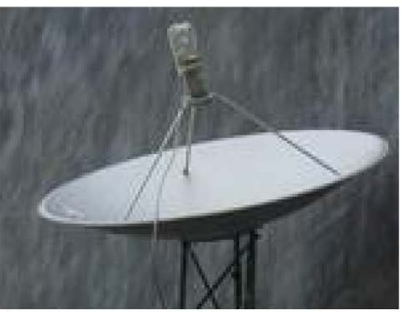

(3) Satellite antennas

Figure 4. TV antennas. 


\section{2) Objective}

The purpose of learning objectives is to provide a clear grasp of the key learning points, as they cannot always maintain their attention in a 45-minute class. Therefore, it is absolutely essential to define the learning objectives at first. Besides, the objectives should be as clear as possible and avoid being too general.

The following objectives for this course are as following: a) Describe the entire procedure from the generation to the reception of the microwave; b) Describe the factors are associated with the received power in the Friis transmission formula. c) Summarize the general rules between the received power and the direction of polarization of the antenna.

The objectives above can avoid common ambiguous terms compared to the words such as "understand", "comprehend", "familiarize" and "master". Thus, students can clearly understand the learning focus of this course.

3) Pre-assessment

The pre-assessment helps teachers know the mastery degree of the students to the knowledge and adjust the teaching focus. It can be conducted through questions, quizzes, brainstorms, and group discussions.

The course is set in the first semester of the senior year. Students have already studied "electromagnetism" or "electrodynamics". Pre-assessment for this course is set to test the students' understanding of the transmission of electromagnetic waves in free space and the direction of antenna polarization. A pre-assessment combination of calling back, question, brainstorm and discussion is adopted by asking questions corresponding to the contents mentioned in Bridge-in as following: Please discuss why TV antenna is improved as it is?

4) Participatory-learning

The significant role of participatory-learning is to increase the engagement of students. Students are expected to use the exploration and discovery thinking to construct knowledge, propose a variety of hypotheses and try to verify them on the issues they are learning, link the current learning content to what they have known, and think critically about the connection. By doing so, we can stimulate the interest of students and help them develop motivation to learn.

Students can be highly engaged in experimental courses. Enlightening teaching links are introduced in this course except the required experimental procedures. It consists of two main sessions so far.

a) Guiding students to use the objects with different shapes and materials in their surroundings to study the wave-absorbing properties. Teachers prepare absorbing materials before the class, such as metal blocks, wooden boards, etc. Figure 5 presents the commonly used absorbing materials. We find that some students with divergent thinking investigate the absorption effect of palms and different shapes of paper with their own mind.

b) We can also study the electromagnetic waveform radiated among communication and wireless network of a phone. The frequencies of China Unicom, China Telecom and China Mobile communications are all in the range between 


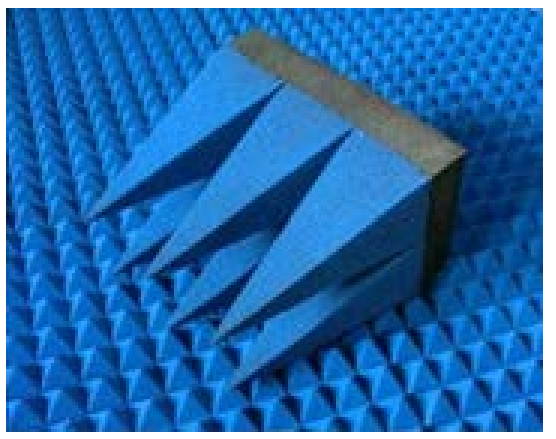

Figure 5. Absorbing materials.

2 and $4 \mathrm{GHz}$. The working band of the horn antennas and high-frequency oscilloscope in the experiment covers this range, too. Thus, the phone can be placed on the horn and then the signals can be directly received by the oscilloscope. In fact, students are very interested in such kind of exploratory research.

5) Post-assessment

We can find out what students have learned and whether they have accomplished learning objectives through post-assessment, which should provide immediate feedback.

The post-assessment for this course includes not only writing experimental reports and answering questions after class, but also a post-assessment session in class which provides immediate feedback from the students. The post-assessment consists of changing the frequency of microwaves produced by the signal source, while keeping the experimental conditions constant.

6) Summary

Summary should be set to summarize the content of the class, help students integrate their learning. We can emphasize on learning objectives in a summary.

In this course, the summary is conducted by asking one student to give a short oral description of the experimental content. Sometimes, the description of students is more appealing to the other students.

\section{Summary}

The BOPPPS instructional model is a student-centered model. In order to improve the instructional performance, we introduce the BOPPPS model into the course "Microwave Transmission and Reception Experiments". During two years of teaching, we find that students are more engaged and more willing to think than that with traditional teaching method. Meanwhile, the authors get a lot of inspiration from the active participation of students. In our follow-up work, we will streamline the design of the content and improve the design of the inspirational sessions.

\section{Conflicts of Interest}

The authors declare no conflicts of interest regarding the publication of this paper. 


\section{References}

Benford, J., Swegle, J. A., \& Schamiloglu, E. (2007). High Power Microwaves (pp. 42-45, 206-207). New York: Taylor \& Francis Group. https://doi.org/10.1201/9781420012064

Gu, M., \& Zhang, K. (1989). Microwave Technology (pp. 62-67). Beijing: Publishing House of Tsinghua University.

Kraus, J. D., \& Marhefka, R. J. (2017). Antennas: For All Applications (pp. 26-27). Chengdu: Publishing House of Electronics Industry. 